\title{
Clinical Significance of the MELF Pattern Myometrial Invasion in Low Grade Endometrioid Type Endometrial Carcinoma
}

\author{
Nejat OZGUL ${ }^{1}$, Fatma Oz ATALAY ${ }^{2}$, Gokhan BOYRAZ¹, Alp USUBUTUN², Utku AKGOR, \\ Murat GULTEKIN $^{1}$, Mehmet Coskun SALMAN ${ }^{1}$ \\ ${ }^{1}$ Hacettepe University, Faculty of Medicine, Division of Gynecologic Oncology \\ ${ }^{2}$ Hacettepe University Faculty of Medicine, Department of Pathology, Ankara, TURKEY
}

\begin{abstract}
The majority of cases with endometrial cancer (EC) have low grade endometrioid type disease which has a favorable prognosis. However, a subgroup of these patients shows a more aggressive clinical course. There are several risk factors defined for the aggressive course including tumor diameter, cervical involvement, depth of myometrial invasion, and lymphovascular space invasion. The morphologic pattern of myometrial invasion was also suggested to be one of the risk factors associated with unfavorable prognosis in these patients. A retrospective study was conducted to investigate the role of microcystic elongated and fragmented (MELF) pattern of myometrial invasion (MI) in patients with low grade endometrioid type EC. Of 276 cases, 69 (25.0\%) had MELF pattern of Ml. The rate of lymph node metastasis was higher in patients with MELF pattern of MI which was an independent risk factor for lymphatic dissemination ( $p<0.001)$. Also, MELF pattern of MI was significantly associated with decreased disease-free survival (DFS) ( $p=0.015)$. In conclusion, in patients with low grade endometrioid type EC, the presence of MELF pattern of MI is associated with the more aggressive clinical course since it is associated with higher rate of lymphatic metastasis and decreased disease-free survival.
\end{abstract}

Keywords: Endometrial cancer, Endometrioid type endometrial cancer, Myometrial invasion, MELF pattern

\section{INTRODUCTION}

Endometrial cancer (EC) is the most common gynecologic malignancy with an estimated 3851 new cases occurring each year in Turkey. ${ }^{1}$ Although many different histologic subtypes of EC exist, the vast majority of EC cases have low grade (International Federation of Gynecology and Obstetrics [FIGO] grade 1 and 2) disease with endometrioid type histology which typically presents at an early stage and have a favorable prognosis. ${ }^{2,3}$ However, a subgroup of patients with low grade endometrioid EC may have a more aggressive clinical course. ${ }^{4,5}$
Several factors such as tumor diameter, cervical involvement, depth of myometrial invasion, and lymphovascular space invasion (LVSI) have been reported for identification of this high-risk subgroup of patients. ${ }^{6-8} \mathrm{In}$ addition to these previously identified risk factors, the morphologic pattern of myometrial invasion (MI) could be one of the risk factors associated with increased risk of extrauterine spread and aggressive clinical behavior among patients with low grade endometrioid EC. . $^{910}$

Endometrioid ECs have several types of MI patterns, including infiltrating glands, broad front, 
adenomyosis-like infiltration, adenoma malignum, and microcystic elongated and fragmented (MELF) pattern. ${ }^{11}$ MELF invasion pattern was first described as the presence of dilation, microcystic change, elongation, and fragmentation of neoplastic glands by Murray in 2003. ${ }^{10}$ Previous studies have shown that MELF pattern invasion is usually detected in low grade endometrioid EC and it could be related to a specific type of tumor-stromal interaction. ${ }^{12,13}$ Moreover, MELF pattern invasion shows not only morphological alteration but also demonstrates an immunophenotypic change including the reduction in hormone receptor and Ecadherin expression, compared with the adjacent glandular tumoral tissue. ${ }^{13}$

Although the clinical significance of MELF pattern of myoinvasion in endometrial cancer is unclear, it has been associated with LVSI and lymph node metastases and suggested to be a potential predictor of extrauterine disease in patients with low grade endometrioid EC. ${ }^{9,10,14-16}$ However, there is an ongoing debate over that issue and prognostic significance of the myoinvasion patterns has not been well established. Therefore, we designed this study to investigate the effect of MELF pattern myoinvasion on lymphatic involvement, risk of recurrence, recurrence pattern (vaginal or distant), and other clinical outcomes including disease-free survival (DFS), and overall survival (OS) in patients with low grade endometrioid EC.

\section{PATIENTS AND METHODS}

Patients with EC who underwent surgical treatment between 2007 and 2016 at Hacettepe University Hospital were specified and clinicopathological data of these patients were retrieved from the computerized database of the Hacettepe University Hospital. Patients who did not undergo complete retroperitoneal lymphadenectomy, who had nonendometrioid histology or stage IV disease and synchronous epithelial ovarian and EC were excluded. Since grade 3 tumors have a more aggressive clinical behaviour when compared to grade 1 and 2 tumors and MELF pattern invasion is usually detected in low grade (grade 1-2) endometrioid EC, cases with grade 3 endometrioid histology were also excluded from the analysis.
All slides of the cases were reviewed by the two gynecologic pathology subspecialists for the presence or absence of MELF pattern MI (Figure 1). Equivocal cases were discussed and a consensus diagnosis was reached. MELF pattern MI was classified as absent, suspicious (number of MELF-like gland focus < 3), or positive (number of MELFlike gland focus $\geq 3$ ). Lymphovascular space invasion was defined as the presence of neoplastic cells within a luminal space of lymphatic channels or a vascular space, and categorized as absent, suspicious ( $<3$ vessels involved), or positive ( $\geq 3$ vessels involved). ${ }^{17,18}$ The primary tumor diameter was considered as the largest diameter of the tumor. Tumor grade was also re-evaluated by using the FIGO grading system that assigned the percentage of solid epithelial component of the tumor.

During the period, among 904 patients with EC, 276 patients constituted our study group who met the following criteria: (1) grade1 and 2 endometrioid-type histology, (2) disease grossly confined to the uterus without preoperative and intraoperative evidence of extra-uterine spread, and (3) had lymphadenectomy (pelvic \pm paraaortic). All patients underwent a total hysterectomy, bilateral salpingooophorectomy, peritoneal washing, and pelvic \pm paraaortic lymphadenectomy. The patients were staged according to the revised 2009 FIGO staging system. The clinical and pathological characteristics of the patients were evaluated, including patient's age, FIGO grade, depth of MI, presence or absence of MELF pattern of myoinvasion, tumor diameter, LVSI, cervical involvement, number of removed lymph nodes, and lymph node metastasis.

All patients entered a routine surveillance program and visits were scheduled for every 3 months for the first 2 years, every 6 months for the next 3 years, and annually thereafter. Surveillance consisted mainly of questioning patients about symptoms and physical examination. Imaging was performed for patients with a suspicion of recurrence during an initial evaluation. The survival status of patients was determined as dead or alive at the time of last follow-up.

\section{Statistical Analysis}

Statistical analyses were performed using Statistical Package for Social Sciences (SPSS) software 


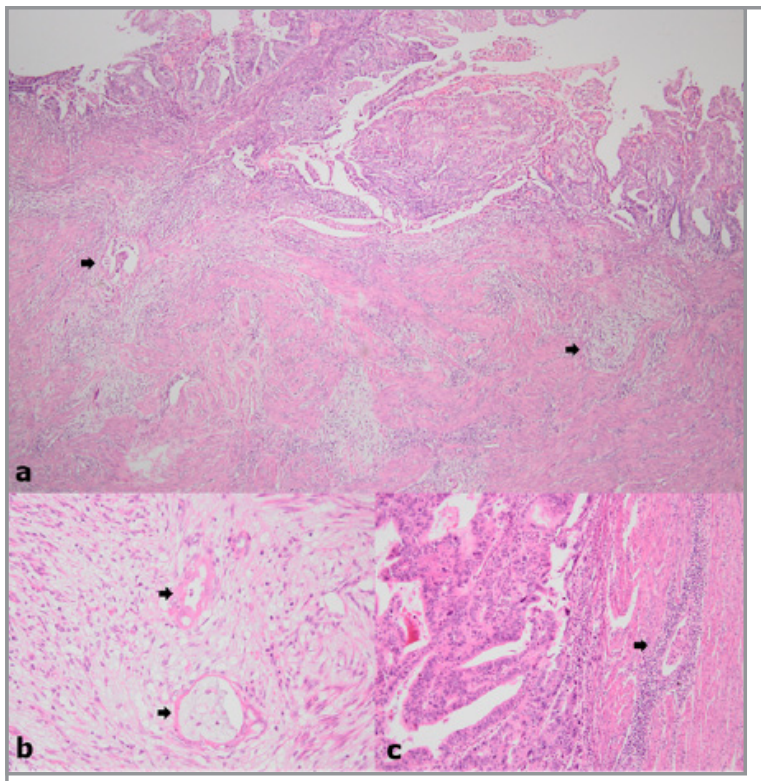

Figure 1. Samples of patients with endometrioid endometrial carcinoma showing MELF pattern of myometrial invasion (arrows). a. MELF pattern (arrows) (HEx40); b. MELF glands (arrows) associated with a fibromyxoid stromal reaction (HEx200); c. Suspicious MELF pattern; presence of MELF-like myometrial invasion pattern only in one gland (arrow) (HEx100)

program (version 22.0). The normality of continuous variable distributions was evaluated using the Kolmogorov-Smirnov test. Categorical variables were evaluated by using the Chi-square test or Fisher's exact test in which appropriate. Non-parametric continuous variables were analyzed using the Mann-Whitney $U$ test. Multivariate analysis was performed by Cox's regression analysis and for the multivariate analysis, the possible factors identified with univariate analysis were further entered into the logistic regression analysis to determine independent predictors of lymphatic involvement. The p-value of $<0.05$ was considered as statistically significant. OS was calculated from the time of diagnosis until death or time of the last follow up. DFS was calculated from the time of diagnosis until the diagnosis of disease recurrence. The Lifetable analysis was used to estimate OS and DFS and survival differences were compared using the Wilcoxon Rank Sum test.

As this study represents a retrospective review, the permission of the Local Ethics Committee was not sought. However, all patients signed an informed consent which allowed our institution to use their clinical data for scientific purposes.

\section{RESULTS}

The mean age of 276 patients was 58.9 years (range; 26-86 years). The demographic and histopathological characteristics of patients are shown in Table 1. Of these cases, 39 (14.1\%) had noninvasive tumors, 69 (25.0\%) had MELF pattern of MI, and 8 (2.9\%) had suspicious MELF pattern. The remaining $168(60.9 \%)$ cases had myometrial invasion without MELF pattern. The LVSI was detected in $38(13.8 \%)$ patients. Although pelvic and paraaortic lymphadenectomies were performed in $221(80.1 \%)$ patients, the remaining 55 (19.9\%) patients had only pelvic lymphadenectomy. Lymph node metastases were found in 24 cases $(8.7 \%)$. Of the 69 patients with MELF pattern of myoinvasion, $45(65.2 \%)$ had grade 1, $24(34.8 \%)$ had grade 2 tumors. Superficial $(\leq 50 \%)$ and deep $(>$ $50 \%)$ MI was detected in $31(45 \%)$ and $38(55 \%)$ patients with MELF pattern of myoinvasion, respectively. The rate of lymph node metastasis was $27.5 \%$ among patients who had MELF pattern of MI, whereas it was only $2.5 \%$ among patients who had no MELF pattern $(\mathrm{p}<0.001)$. In other words, among the 24 patients with lymph node metastasis, 19 (79.1\%) had MELF pattern of MI, and the remaining $5(20.9 \%)$ had no MELF pattern. Similarly, patients with myometrial invasion $(n=237)$ revealed higher rates of lymph node metastasis when MELF pattern of MI was detected $(3.1 \%$ vs. $27.5 \%, \mathrm{p}<0.001)$. In addition to MELF pattern of MI, tumor size, deep myometrial invasion, cervical stromal involvement, and the presence of LVSI were found to be significant predictors of lymphatic spread in univariate analysis (Table 2).

Multivariate logistic regression analysis was performed to identify factors related to lymphatic involvement. The examined factors included age, myometrial invasion, cervical stromal involvement, LVSI, tumor size, and MELF pattern of MI. MELF pattern of MI (OR: 9.36, 95\% CI: 2.9$36.21, \mathrm{p}=0.001)$, cervical stromal involvement (OR: $7.20,95 \%$ CI: $1.84-28.15, \mathrm{p}=0.005$ ), LVSI (OR: $3.77,95 \%$ CI: $1.6-10.58, \mathrm{p}=0.03$ ), and tumor size (OR: $0.63,95 \% \mathrm{CI}: 0.44-0.89, \mathrm{p}=0.01$ ) were the independent predictors associated with lymph node metastasis. 


\begin{tabular}{|c|c|}
\hline Characteristic & $\begin{array}{l}\text { Entire cohort } \\
n=276\end{array}$ \\
\hline Age (years) ${ }^{a}$ & $58.9 \pm 10.2$ \\
\hline \multicolumn{2}{|l|}{ FIGO Grade ${ }^{b}$} \\
\hline Grade I & 207 (75\%) \\
\hline Grade ॥ & $69(25 \%)$ \\
\hline \multicolumn{2}{|l|}{ Myometrial invasion ${ }^{\mathrm{b}}$} \\
\hline None & $39(14.1 \%)$ \\
\hline$<50 \%$ & $150(54.3 \%)$ \\
\hline$\geq 50 \%$ & 87 (31.6\%) \\
\hline \multicolumn{2}{|l|}{ Cervical stromal involvement ${ }^{\mathrm{b}}$} \\
\hline Yes & $32(11.6 \%)$ \\
\hline No & $244(88.4 \%)$ \\
\hline \multicolumn{2}{|l|}{ Lymphovascular space invasion ${ }^{b}$} \\
\hline Yes & $38(13.8 \%)$ \\
\hline No & $231(83.7 \%)$ \\
\hline Suspicious & $7(2.5 \%)$ \\
\hline \multicolumn{2}{|l|}{ MELF pattern } \\
\hline Yes & $69(25 \%)$ \\
\hline No & $199(72.1 \%)$ \\
\hline Suspicious & 8 (2.9\%) \\
\hline \multicolumn{2}{|l|}{ Primary tumor size $(\mathrm{cm})^{b}$} \\
\hline$\leq 2 \mathrm{~cm}$ & $63(22.8 \%)$ \\
\hline$>2 \mathrm{~cm}$ & $213(77.2 \%)$ \\
\hline \multicolumn{2}{|l|}{ Lymphadenectomy procedure ${ }^{\mathrm{b}}$} \\
\hline Only pelvic & 55 (19.9\%) \\
\hline Pelvic and paraaortic & 221 (80.1\%) \\
\hline Number of lymph nodes retrievedc & $37(5-128)$ \\
\hline Pelvic lymph nodes & $24(4-78)$ \\
\hline Paraaortic lymph nodes & $12(5-65)$ \\
\hline \multicolumn{2}{|l|}{ Lymph node metastasis } \\
\hline Yes & $24(8.7 \%)$ \\
\hline No & $252(91.3 \%)$ \\
\hline \multicolumn{2}{|l|}{ Stage at diagnosis } \\
\hline Stage I & $230(83.3 \%)$ \\
\hline Stage II & $15(5.4 \%)$ \\
\hline Stage III & $31(11.2 \%)$ \\
\hline \multicolumn{2}{|l|}{ Recurrencesb } \\
\hline None & 261 (94.6\%) \\
\hline Vaginal vault & 7 (2.5\%) \\
\hline Distant & $8(2.9 \%)$ \\
\hline \multicolumn{2}{|c|}{$\begin{array}{l}\text { a Numerical variables are expressed as mean } \pm \text { standart deviation (SD } \\
\text { b Categorical variables are expressed as number (\%) } \\
\text { ' Numerical variables are expressed as median (min-max) }\end{array}$} \\
\hline
\end{tabular}

Adjuvant treatment decisions for all patients were made by a multidisciplinary tumor board. Of the 276 patients, 167 patients $(60.5 \%)$ did not receive adjuvant therapy. Thirty-five patients (12.7\%) had adjuvant therapy that included pelvic external beam radiotherapy and platinum-based chemotherapy. The remaining 74 patients $(26.8 \%)$ had vaginal brachytherapy.

Median follow-up was 40 months (range; 3-151 months). The 5-year DFS for the entire cohort was $92 \%$. Disease recurred in 15 patients $(5.4 \%)$. The characteristics of patients with recurrent disease are summarized in Table 3. Seven (46.6\%) patients had an isolated vaginal vault recurrence and $8(53.4 \%)$ had a distant recurrence. The median time to recurrence was 16 months (range; 7-57 months). Of the 15 patients with recurrent disease, 7 (46.7\%) had MELF pattern, 7 (46.7\%) had no MELF pattern, and the remaining $1(6.6 \%)$ had suspicious MELF pattern of MI. Among 7 patients with isolated vaginal vault recurrence, 2 (28.6\%) had MELF pattern and $4(57.1 \%)$ had no MELF pattern of MI. The remaining one patient had a suspicious MELF pattern. On the other hand, of the 8 patients with distant recurrences, $5(62.5 \%)$ patients had MELF pattern of MI and $3(37.5 \%)$ had no MELF pattern of MI. In other words, among 7 recurrent patients with MELF pattern of MI, 2 (28.6\%) had vaginal and $5(71.4 \%)$ had distant recurrences. Among 7 non-MELF recurrent patients, $4(57.1 \%)$ had vaginal recurrences and $3(42.9 \%)$ had distant recurrences. Distant recurrences were found to be high among the patients who had MELF pattern of MI (71.4\%) when compared to the patients who had no MELF pattern of MI (42.9\%). However, the difference was not statistically significant $(\mathrm{p}=0.09)$.

The 5-year OS was $94 \%$. After a median followup of 40 months, 6 patients died from their disease and 8 patients died of other causes. The 5 year DFS was $\% 86$ among patients with MELF pattern of MI whereas it was $96 \%$ among non-MELF cases ( $\mathrm{p}=$ 0.015, HR: 3.51, 95\% CI: 1.37-9.69). Accordingly, MELF pattern of MI was significantly associated with decreased DFS (Figure 2). However, there was no significant difference in 5 years OS between MELF and non-MELF cases (89\% vs 96\%, $\mathrm{p}=0.16)($ Figure 3$)$. 
International Journal of Hematology and Oncology

\begin{tabular}{|c|c|c|c|}
\hline Characteristic & $\begin{array}{l}\text { Positive Lymph Nodes } \\
n=24\end{array}$ & $\begin{array}{l}\text { Negative Lymph Nodes } \\
\mathrm{n}=252\end{array}$ & $p$ value \\
\hline Age (years) ${ }^{a}$ & $61.1 \pm 9.52$ & $58.7 \pm 10.29$ & 1.0 \\
\hline$>60$ & $10(8.7 \%)$ & $105(91.3 \%)$ & \\
\hline$\leq 60$ & $14(8.7 \%)$ & $147(91.3 \%)$ & \\
\hline \multicolumn{4}{|l|}{ FIGO grade } \\
\hline Grade I & 8 (8.6\%) & 189 (91.4\%) & 1.0 \\
\hline Grade ॥ & $6(8.6 \%)$ & $63(91.4 \%)$ & \\
\hline Primary tumor size $(\mathrm{cm})^{b}$ & $5(2.8)$ & $3(1.8)$ & 0.001 \\
\hline \multicolumn{4}{|l|}{ Myometrial invasion } \\
\hline$<50 \%$ & $8(4.2 \%)$ & $181(95.8 \%)$ & $<0.001$ \\
\hline$\geq 50 \%$ & $16(18.4 \%)$ & $71(81.6 \%)$ & \\
\hline \multicolumn{2}{|l|}{ Lymphovascular space invasion } & $24(63.2 \%)$ & \\
\hline Yes & $14(36.8)$ & 221 (95.7\%) & $<0.001$ \\
\hline No & $10(4.3 \%)$ & 7 (100\%) & \\
\hline Suspicious & $0(0 \%)$ & & \\
\hline \multicolumn{2}{|l|}{ Cervical stromal invasion } & $20(62.5 \%)$ & \\
\hline Yes & $12(37.5 \%)$ & 232 (95.1\%) & $<0.001$ \\
\hline No & 12 (4.9\%) & & \\
\hline Number of retrieved lymph nodes ${ }^{b}$ & $38(32)$ & $31(22)$ & 0.055 \\
\hline \multicolumn{4}{|l|}{ MELF pattern of myometrial invasion } \\
\hline Yes & $19(27.5 \%)$ & 50 (72.5\%\%) & $<0.001$ \\
\hline No & $5(2.5 \%)$ & $194(97.5 \%)$ & \\
\hline Suspicious & $0(0 \%)$ & $8(100 \%)$ & \\
\hline \multicolumn{4}{|c|}{$\begin{array}{l}\text { Bold text indicates a statistically significant difference with a } p<0.05 \\
\text { a Values are expressed as mean (range) } \\
{ }^{b} \text { Values are expressed as median (IQ range). Statistical analysis was performed using Mann-Whitney } \cup \text { test }\end{array}$} \\
\hline
\end{tabular}

\section{DISCUSSION}

An important and current question is why a subgroup of patients with low grade endometrioid EC have more aggressive clinical behaviour than others? Based on this question, there is an ongoing debate about whether MELF pattern of myometrial invasion is a risk factor for the extrauterine spread and aggressive clinical behavior among patients with low grade endometrioid EC. Therefore, the present study was conducted among 276 patients with grade 1 and 2 endometrioid EC, with particular focus on the clinical significance of MELF pattern of MI.

More recent studies estimated the rate of MELF pattern of MI in patients with low grade endome- trioid EC at $10-34 \% .{ }^{19,20}$ Consistent with the literature, MELF pattern of MI was detected in 25\% of patients with grade 1-2 endometriod EC in the present study. In addition, while MELF pattern of MI was detected in $79.1 \%$ of patients with lymphatic involvement, only $19.8 \%$ of patients without lymphatic involvement had MELF invasion. Consequently, according to our study, compared to patients without MELF pattern of MI those with MELF pattern had significantly higher rates of lymphatic metastasis $(2.5 \%$ vs. $27.5 \%, \mathrm{p}<0.001)$, and MELF pattern of MI was detected as an independent predictor of lymph node metastasis in patients with grade 1 and 2 endometrioid EC. Similarly, Sanci et al. and Altunpulluk et al. showed 


\begin{tabular}{|c|c|c|c|c|c|c|c|c|c|c|c|c|}
\hline & $\begin{array}{l}\text { Age } \\
\text { (y) }\end{array}$ & MELF & $\begin{array}{l}\text { Depth } \\
\text { of } \mathrm{MI}\end{array}$ & $\begin{array}{l}\text { Tumor } \\
\text { size } \\
\text { (cm) }\end{array}$ & LVSI & $\begin{array}{l}\text { Lymph } \\
\text { node } \\
\text { metastasis }\end{array}$ & $\begin{array}{l}\text { Cervical } \\
\text { stromal } \\
\text { involveme }\end{array}$ & $\begin{array}{l}\text { FIGO } \\
\text { stage } \\
\text { ent }\end{array}$ & $\begin{array}{l}\text { Site of } \\
\text { Recurrence }\end{array}$ & $\begin{array}{l}\text { DFS } \\
\text { (m) }\end{array}$ & Status & $\begin{array}{l}\text { OS } \\
\text { (m) }\end{array}$ \\
\hline 1 & 65 & $\mathrm{P}$ & $>50 \%$ & 6 & $\mathrm{~N}$ & $\mathrm{~N}$ & $\mathrm{~N}$ & $1 \mathrm{~B}$ & Vaginal & 8 & exitus* & 18 \\
\hline 2 & 65 & $\mathrm{P}$ & $>50 \%$ & 3.5 & $\mathrm{~N}$ & $\mathrm{~N}$ & $\mathrm{~N}$ & $1 \mathrm{~B}$ & Vaginal & 57 & alive & 84 \\
\hline 3 & 37 & $P$ & $>50 \%$ & 2.5 & $\mathrm{~N}$ & $\mathrm{~N}$ & $\mathrm{~N}$ & $1 \mathrm{~B}$ & Pelvic & 16 & exitus & 22 \\
\hline 4 & 65 & $P$ & $>50 \%$ & 3 & $\mathrm{~N}$ & $\mathrm{~N}$ & $\mathrm{~N}$ & $1 \mathrm{~B}$ & Pelvic & 66 & alive & 73 \\
\hline 5 & 59 & $\mathrm{P}$ & $>50 \%$ & 2 & $\mathrm{P}$ & $P$ & $\mathrm{P}$ & $3 \mathrm{C} 2$ & Abdomen & 12 & exitus & 17 \\
\hline 6 & 73 & $\mathrm{P}$ & $>50 \%$ & 8 & $P$ & $P$ & $P$ & $3 \mathrm{C} 2$ & Abdomen & 10 & exitus & 26 \\
\hline 7 & 58 & $\mathrm{P}$ & $>50 \%$ & 5 & $\mathrm{~N}$ & $P$ & $\mathrm{~N}$ & $3 \mathrm{C} 1$ & Lung & 11 & exitus & 23 \\
\hline 8 & 68 & $\mathrm{~N}$ & $>50 \%$ & 4 & $P$ & $\mathrm{~N}$ & $\mathrm{~N}$ & $1 \mathrm{~B}$ & Vaginal & 10 & alive & 15 \\
\hline 9 & 75 & $\mathrm{~N}$ & $>50 \%$ & 5 & $\mathrm{~N}$ & $\mathrm{~N}$ & $\mathrm{~N}$ & $1 \mathrm{~B}$ & Vaginal & 11 & alive & 21 \\
\hline 10 & 74 & $\mathrm{~N}$ & $>50 \%$ & 5.5 & $\mathrm{~N}$ & $\mathrm{~N}$ & $\mathrm{~N}$ & $1 \mathrm{~B}$ & Vaginal & 28 & alive & 38 \\
\hline 11 & 60 & $\mathrm{~N}$ & $>50 \%$ & 3.5 & $P$ & $P$ & $\mathrm{P}$ & $3 \mathrm{C} 1$ & Pelvic & 8 & alive & 37 \\
\hline 12 & 81 & $\mathrm{~N}$ & $>50 \%$ & 5 & $\mathrm{~N}$ & $\mathrm{~N}$ & $\mathrm{P}$ & 2 & Liver & 26 & exitus & 34 \\
\hline 13 & 63 & $\mathrm{~N}$ & $\leq 50 \%$ & 2.5 & N & N & $\mathrm{N}$ & $1 \mathrm{~A}$ & Vaginal & 62 & alive & 83 \\
\hline 14 & 50 & $\mathrm{~N}$ & $\leq 50 \%$ & 8 & $\mathrm{~N}$ & $P$ & $P$ & $3 \mathrm{C} 1$ & Liver & 17 & exitus & 27 \\
\hline 15 & 61 & S & $>50 \%$ & 3.5 & $\mathrm{~N}$ & N & $\mathrm{N}$ & $1 \mathrm{~B}$ & Vaginal & 30 & alive & 69 \\
\hline \multicolumn{13}{|c|}{$\begin{array}{l}y=\text { years; } m=\text { months; } M E L F=\text { Microcystic elongated and fragmented, } M I=\text { Myometrial invasion, } L V S I=L y m p h o v a s c u l a r \text { space invasion, } \\
D F S=\text { Disease-free survival; OS: Overall survival; } P=\text { positive; } N=\text { negative; } S: \text { suspicious }\end{array}$} \\
\hline Exit! & . & who die & diseas & * & s who & d of tho & wn causes & & & & & \\
\hline
\end{tabular}

that MELF pattern of MI was an independent predictor of lymphatic involvement in multivariate analysis. ${ }^{20}$ On the other hand, although Euscher et al. and Han et al. demonstrated that MELF pattern of MI was an important risk factor for lymphatic involvement in univariate analysis, they failed to demonstrate any association between MELF pattern of MI and lymphatic involvement in multivariate analysis. ${ }^{14}$ Stewart et al. reported that lymph node metastasis was detected in $56 \%$ of patients who had MELF pattern of MI and in 7\% of those who had not. ${ }^{13}$ Pavlakis et al. found similar results showing that the lymphatic involvement rate was $53.8 \%$ among the MELF cases whereas it was only $6.9 \%$ among non-MELF cases. ${ }^{15}$ Another important study for significance of MELF pattern MI in patients with EC was published by Joehlin-Price et al. ${ }^{21}$ They evaluated 464 patients with grade 1 endometrioid EC and MELF pattern of MI was detected in $12.9 \%$ of their patients. Among the patients with lymphatic involvement, the proportions of MELF case and non-MELF cases were
$18.3 \%$ and $1.2 \%$, respectively $(\mathrm{p}<0.001)$. In addition to association between MELF pattern of MI and lymph node metastasis, they also evaluated the recurrence pattern in patients with and without MELF pattern. They reported that 20 patients $(4.3 \%)$ developed recurrent disease (9 isolated vaginal and 11 distant recurrences). Interestingly, none of the patients with MELF pattern of MI had isolated vaginal recurrences, and MELF cases had a tendency for extravaginal distant recurrences, but the difference was not statistically significant. However, in the present study, among 7 patients with isolated vaginal vault recurrence, $2(28.6 \%)$ had MELF pattern of MI. Also, similar to study by Joehlin-Price et al., distant recurrences were found to be higher among patients who had MELF pattern of MI (71.4\%) when compared to the patients who had no MELF pattern (42.9\%) in the present study. However, the difference was not statistically significant. On the other hand, Maschiano et al. found that MELF pattern of MI was identified in $53 \%$ of patients with distant recurrences, but only 


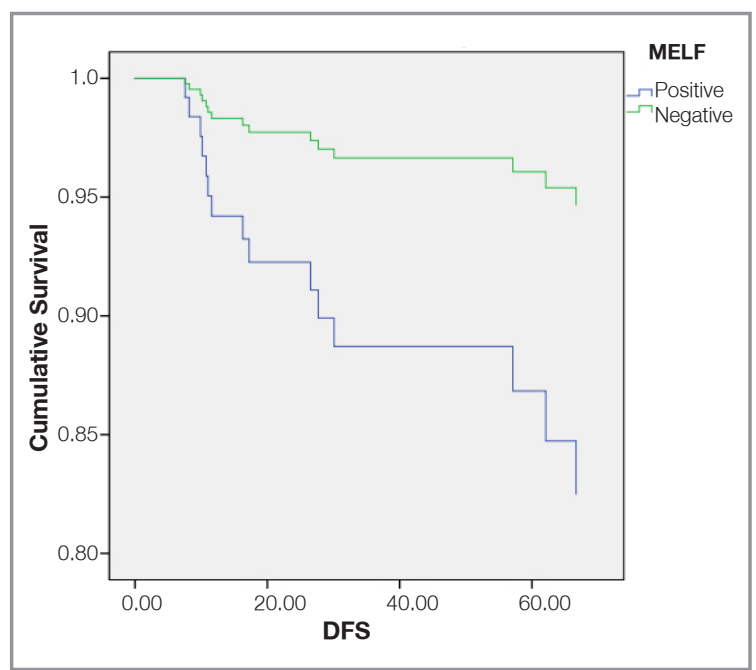

Figure 2. Life-table analysis showing significant association of MELF pattern with disease-free survival

in $30 \%$ of those with vaginal vault recurrences and the difference was statistically significant. ${ }^{22}$

Currently, there is limited information in the literature related to the effect of MELF pattern MI on oncological outcomes including OS and DFS. Interestingly, Sanci et al. reported that although MELF pattern of MI was an independent prognostic factor for lymphatic metastasis, it was significantly associated with increased OS, and there was no significant difference in DFS between MELF and non-MELF cases. ${ }^{20}$ In contrast, according to our results, MELF pattern of MI was significantly associated with decreased DFS, and no statistically significant OS difference was detected between MELF and non-MELF cases. On the other hand, Kihara et al. reported that MELF pattern of MI affected neither disease-specific survival nor DFS. ${ }^{23}$ These differences can be associated with a small number of patients, retrospective nature of the studies, and differences in study design.

Although sentinel lymph node mapping is becoming an increasingly popular approach in the surgical treatment of endometrial cancer, it has some limitations such as cost and availability of technical infrastructure, and also there is not enough evidence at the present time to support its routine use. Therefore, we use Mayo risk stratification models for predicting lymphatic dissemination in patients with endometrioid EC, which is still the most

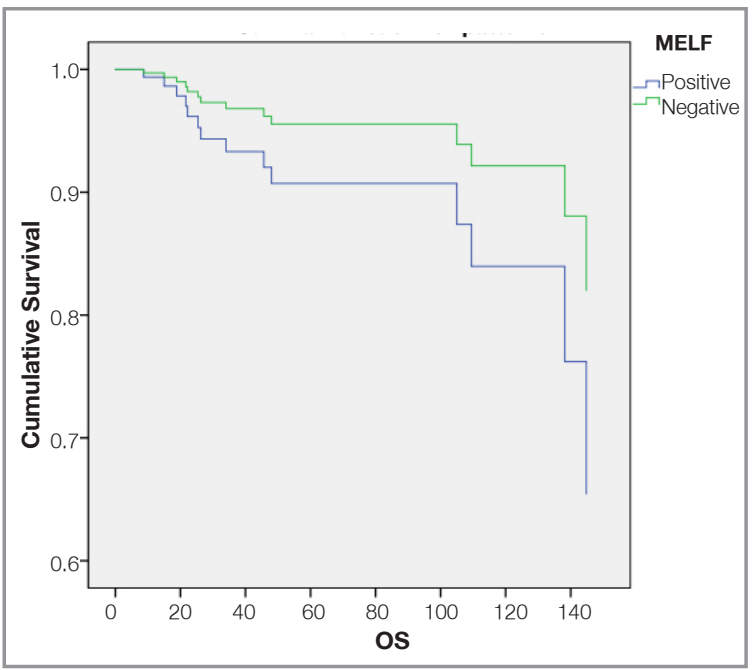

Figure 3. Life-table analysis showing similar overall survival between MELF and non-MELF cases

widely model for this purpose. ${ }^{24}$ This risk model is mainly based on tumor grade, histologic type, tumor diameter, and MI. However, MELF pattern of MI is not considered as a risk factor for extrauterine spread in conventional risk stratification models. In the light of these findings, we suggest that MELF pattern of MI could be used in addition to these traditional risk factors during the pathologic evaluation to predict lymph node metastasis. In addition, combination of traditional risk factors with MELF pattern of MI could be beneficial for management strategies in the postoperative period in patients who are incidentally diagnosed to have EC after hysterectomy done for benign conditions. Evaluation of MELF pattern of MI during intraoperative frozen section is not a common practice and identification of this pattern would be difficult in frozen sections which usually have severe artifacts compared to paraffin section. However, if the presence of MELF pattern may be detected during intraoperative evaluation in patients with low risk factors according to current risk stratification models, this may influence clinical decision making on whether to perform lymphadenectomy.

Our study has inherent limitations due to its retrospective nature. The presence of other possible confounding variables such as selection and recall bias that might have affected our results could not be ruled out. Another limitation of our study was 
the small sample size. Despite these limitations, our study adds significantly to the existing literature and to our knowledge this study has one of the largest series in the literature regarding the importance of MELF pattern of MI in patients with low grade endometrioid EC based on a pathological review.

\section{Conclusion}

MELF pattern of MI was an independent predictor of lymph node metastasis in patients with grade1 and 2 endometrioid EC. Therefore, we recommend that the presence of MELF pattern of MI should be noted in the pathology reports in patients with endometriod EC. Although MELF pattern of MI was significantly associated with decreased DFS, no differences were observed between MELF and non-MELF cases with regard to OS. Moreover, even though there was a trend of having higher distant recurrence rates in MELF cases, the difference did not reach statistical significance and further studies with a larger sample size are required to demonstrate this relationship.

\section{REFERENCES}

1. Gultekin M, Kucukyildiz I, Karaca MZ, et al. Trends of Gynecological Cancers in Turkey: Toward Europe or Asia? Int J Gynecol Cancer 27: 1525-1533, 2017.

2. Creasman WT, Odicino F, Maisonneuve P, et al. Carcinoma of the corpus uteri. FIGO 26th Annual Report on the Results of Treatment in Gynecological Cancer. Int J Gynaecol Obstet 95 Suppl 1: S105-143, 2006.

3. Group SGOCPECW, Burke WM, Orr J, et al. Endometrial cancer: a review and current management strategies: part I. Gynecol Oncol 134: 385-392, 2014.

4. Nugent EK, Bishop EA, Mathews CA, et al. Do uterine risk factors or lymph node metastasis more significantly affect recurrence in patients with endometrioid adenocarcinoma? Gynecol Oncol 125: 94-98, 2012.

5. Group SGOCPECW, Burke WM, Orr J, et al. Endometrial cancer: a review and current management strategies: part II. Gynecol Oncol 134: 393-402, 2014.

6. Boyraz G, Salman MC, Gultekin M, et al. Incidence of Lymph Node Metastasis in Surgically Staged FIGO IA G1/G2 Endometrial Cancer With a Tumor Size of More Than $2 \mathrm{~cm}$. Int J Gynecol Cancer 27: 486-492, 2017.

7. Schink JC, Rademaker AW, Miller DS, et al. Tumor size in endometrial cancer. Cancer 67: 2791-2794, 1991.
8. Madom LM, Brown AK, Lui F, et al. Lower uterine segment involvement as a predictor for lymph node spread in endometrial carcinoma. Gynecol Oncol 107: 75-78, 2007.

9. Han G, Lim D, Leitao MM, Jr., et al. Histological features associated with occult lymph node metastasis in FIGO clinical stage I, grade I endometrioid carcinoma. Histopathology 64: 389-398, 2014.

10. Murray SK, Young RH, Scully RE. Unusual epithelial and stromal changes in myoinvasive endometrioid adenocarcinoma: a study of their frequency, associated diagnostic problems, and prognostic significance. Int J Gynecol Pathol 22: 324-33, 2003.

11. Quick CM, May T, Horowitz NS, et al. Low-grade, low-stage endometrioid endometrial adenocarcinoma: a clinicopathologic analysis of 324 cases focusing on frequency and pattern of myoinvasion. Int J Gynecol Pathol 31: 337-343, 2012.

12. Stewart CJ, Little L. Immunophenotypic features of MELF pattern invasion in endometrial adenocarcinoma: evidence for epithelial-mesenchymal transition. Histopathology 55: 91101, 2009.

13. Stewart CJ, Brennan BA, Leung YC, et al. MELF pattern invasion in endometrial carcinoma: association with low grade, myoinvasive endometrioid tumours, focal mucinous differentiation and vascular invasion. Pathology 41: 454-459, 2009.

14. Euscher E, Fox P, Bassett R, et al. The pattern of myometrial invasion as a predictor of lymph node metastasis or extrauterine disease in low-grade endometrial carcinoma. Am J Surg Pathol 37: 1728-1736, 2013.

15. Pavlakis K, Messini I, Vrekoussis T, et al. MELF invasion in endometrial cancer as a risk factor for lymph node metastasis. Histopathology 58: 966-973, 2011.

16. Hertel JD, Huettner PC, Pfeifer JD. Lymphovascular space invasion in microcystic elongated and fragmented (MELF)pattern well-differentiated endometrioid adenocarcinoma is associated with a higher rate of lymph node metastasis. Int $\mathrm{J}$ Gynecol Pathol 33: 127-134, 2014.

17. Bosse $T$, Peters EE, Creutzberg CL, et al. Substantial lymphvascular space invasion (LVSI) is a significant risk factor for recurrence in endometrial cancer- A pooled analysis of PORTEC 1 and 2 trials. Eur J Cancer 51: 1742-1750, 2015.

18. McCluggage WG. Pathologic Staging of Endometrial Carcinomas: Selected Areas of Difficulty. Adv Anat Pathol 25: 71 84, 2018.

19. Stewart CJ, Crook ML, Manso L. Fascin expression in lowgrade uterine endometrioid adenocarcinoma: correlation with microcystic, elongated and fragmented (MELF)-type alteration at the deep invasive margin. Histopathology 59: 73-80, 2011.

20. Sanci M, Gungorduk K, Gulseren V, et al. MELF Pattern for Predicting Lymph Node Involvement and Survival in Grade I-II Endometrioid-type Endometrial Cancer. Int J Gynecol Pathol 37: 17-21, 2018.

21. Joehlin-Price AS, McHugh KE, Stephens JA, et al. The Microcystic, Elongated, and Fragmented (MELF) Pattern of Invasion: A Single Institution Report of 464 Consecutive FIGO Grade 1 Endometrial Endometrioid Adenocarcinomas. Am J Surg Pathol 41: 49-55, 2017. 
22. Moschiano EJ, Barbuto DA, Walsh C, et al. Risk factors for recurrence and prognosis of low-grade endometrial adenocarcinoma; vaginal versus other sites. Int J Gynecol Pathol 33: 268-273, 2014.

23. Kihara A, Yoshida $H$, Watanabe $R$, et al. Clinicopathologic Association and Prognostic Value of Microcystic, Elongated, and Fragmented (MELF) Pattern in Endometrial Endometrioid Carcinoma. Am J Surg Pathol 41: 896-905, 2017.

24. Mariani A, Webb MJ, Keeney GL, et al. Low-risk corpus cancer: is lymphadenectomy or radiotherapy necessary? Am J Obstet Gynecol 182: 1506-1519, 2000.

\section{Correspondence:}

Dr. Utku AKGOR

Hacettepe Universitesi Tip Fakultesi

Kadin Hastaliklari ve Dogum Anabilim Dali

06100, Sihhiye, Ankara, TURKEY

Tel: (+90-312) 3053128

Fax: (+90-312) 3116372

e-mail: utkuakgor@gmail.com

\section{ORCIDs:}

Nejat Ozgul

Fatma Oz Atalay

Gokhan Boyraz

Alp Usubutun

Utku Akgor

Murat Gultekin

Mehmet Coskun Salman
0000-0002-4257-9731

0000-0002-7188-6115

0000-0001-6165-1497

$0000-0001-9572-7875$

0000-0003-1377-2651

0000-0002-4221-4459

0000-0003-1504-1756 